\title{
TWICE PERIODIC MEASURABLE FUNCTIONS
}

\author{
Abstract \\ In this note we prove that, for $a, b \in(0,1)$ and $f$ a measurable \\ function mapping $[0,1]$ to $\mathbb{R}$, the following statements are equivalent: \\ (i) $f(x)=f(x-a)$ a.e. in $[a, 1]$ and $f(x)=f(x-b)$ a.e. in $[b, 1]$ \\ implies that $f$ is a.e. constant in $[0,1]$. \\ (ii) $a+b \leq 1$ and $a / b$ is irrational.
}

Dealing with periods of measurable functions it is well known that, for a periodic real-valued function defined on $\mathbb{R}$, either there exists the smallest positive period $t_{0}$ and all periods are of the form $n t_{0}$ where $n$ is any integer, or the set of the periods is dense. Moreover, if a measurable function has a dense set of periods then it is a.e. constant. On the other hand, if a twice periodic measurable real-valued function is defined on the interval $[0,1]$, no results like the above, imposing conditions on the periods of the function, seem to exist in the literature.

Denote by $\mathcal{F}_{a}$ the set of measurable functions $f:[0,1] \rightarrow \mathbb{R}$ such that $f(x)=f(x-a)$ a.e. in $[a, 1]$ where $a \in(0,1)$, and let $\mathcal{C}$ be the set of functions mapping $[0,1]$ to $\mathbb{R}$ which are constant a.e. in $[0,1]$. The result we shall prove is the following:

Theorem 1. If $a, b \in(0,1)$ then $a+b \leq 1$ and $a / b$ is irrational $\Leftrightarrow \mathcal{F}_{a} \cap \mathcal{F}_{b}=\mathcal{C}$.

Let us first prove an auxiliary result. For any function $f:[0,1] \rightarrow \mathbb{R}$ let $H_{f}$ be the set of points $a \in(0,1)$ such that $f(x)=f(x-a)$ a.e. in $[a, 1]$.

Lemma 2. Let $f:[0,1] \rightarrow \mathbb{R}$ and suppose $a, b \in H_{f}, a+b \leq 1$, and $a / b$ is irrational. Then $H_{f}$ is dense in $[0,1]$.

Key Words: periodic measurable functions, Lebesgue density theorem

Mathematical Reviews subject classification: 28A20

Received by the editors May 28, 1998 
Proof. Assume $a<b$. Let $a_{0}:=a, b_{0}:=b$ and define recursively $a_{n+1}:=$ $\min \left\{a_{n}, b_{n}-a_{n}\right\}$ and $b_{n+1}:=\max \left\{a_{n}, b_{n}-a_{n}\right\}(n \in \mathbb{N} \cup\{0\})$. Let us show that $a_{n} \rightarrow 0, n \rightarrow \infty$. The fact that the limits of $a_{n}$ and $b_{n}$ exist and are nonnegative follows since the sequences are decreasing and nonnegative. If $x=\lim a_{n}$ and $y=\lim b_{n}$, using that $a_{n}+b_{n}=b_{n-1}$ we get

$$
x+y=\lim _{n \rightarrow \infty}\left(a_{n}+b_{n}\right)=\lim _{n \rightarrow \infty} b_{n-1}=y
$$

implying that $x=0$. Now, one can easily show that $\left\{a_{n}\right\} \subset H_{f}$ which in turn implies (we omit the trivial proof) that $H_{f}$ is dense.

PROOF OF THEOREM.

" $\Rightarrow$ ": Suppose $f:[0,1] \rightarrow \mathbb{R}$ measurable is not a.e. constant but $a, b \in H_{f}$, $a+b \leq 1$ and $a / b$ is irrational. Then the inverse image of some interval is a set $A$ with measure $0<m(A)<1$. By the Lebesgue density theorem there is an interval $I$ (with length less than $\epsilon>0$ ) where the density of $A$ is less than $\epsilon$. If $h$ is in $H_{f}$ and $I+h$ (or $\left.I-h\right)$ is in $(0,1)$ then the intersection of $A$ and $I$ is congruent to the intersection of $A$ and $I+h$ (or $I-h$ ), so the density is the same in $I+h$ (or $I-h$ ). By lemma, $H_{f}$ is dense, and so we can cover almost the whole $(0,1)$ interval (with an exception of finitely many intervals with total length less than $\epsilon$ ) with disjoint translates using translations from $H_{f}$. Since in each translate the density is less than $\epsilon$ and only less than $\epsilon$ is uncovered we get that $m(A)<2 \epsilon$ for any $\epsilon>0$, which is a contradiction.

" $\Leftarrow$ ": Suppose $0<a<b<1$ are such that $a+b>1$ (the $a / b$ rational case is quite obvious). Let $A_{0}:=[1-b, a)$. If $A_{k}$ is in $[0,1-a)$ then let $A_{k+1}:=A_{k}+a$; if $A_{k}$ is in $[b, 1)$ then let $A_{k+1}:=A_{k}-b$; if neither then let $m:=k$ and stop. It is easily seen that if $x \in A_{i} \cap A_{j}(i<j)$ then either $x-a$ or $x+b$ is in $A_{i-1}$ and $A_{j-1}$. Repeating this, we get that $A_{j-i}$ intersects $A_{0}=[1-b, a)$ which cannot happen by definition. Thus $A_{0}, A_{1}, \ldots$ are disjoint intervals with length $a+b-1>0$ and so $m$ must be finite. Let $B_{m}$ be a proper subinterval of the intersection of $A_{m}$ and $[1-a, b)$ and, going backwards, define $B_{k}$ as a subinterval of $A_{k}$ such that $B_{k+1}=B_{k}+a$ or $B_{k}-b$ in the same way as in the definition of $A_{k}$. Then the characteristic function of the union of $B_{0}, B_{1}, \ldots, B_{m}$ is in $\mathcal{F}_{a}$ and $\mathcal{F}_{b}$ but not a.e. constant.

Acknowledgement. The authors wish to thank the referee who simplified considerably the previous proof of the main result. 